\title{
The role of safe work method statements in the Australian construction industry
}

\author{
Author: \\ David Borys \\ Affiliation(s) Description/Correspondence: \\ School of Health Sciences, University of Ballarat, VIC, Australia \\ +61353279159. \\ E-mail address: d.borys@ballarat.edu.au
}

\begin{abstract}
:
The aim of this study was to determine how managers and workers interpret and use safe work method statements (SWMS) in the Australian construction industry in order to explore if there was a gap between work as imagined and work as performed. Despite recent improvements in its safety performance, the Australian construction industry continues to be among the top four most dangerous industries within which to work. SWMS are a key strategy relied upon by the construction industry to reduce this toll. However, few, if any studies have looked at the role of SWMS in creating a safe workplace. This ethnographic study focused on the role of SWMS at two commercial construction sites in Australia. The researcher spent six months 'getting to know' the organisation before conducting 18 semistructured interviews spanning labourers, supervisors and managers. The researcher also collected examples of completed SWMS. This study found: (1) that SWMS are important for safety, particularly for tasks that are out of the ordinary; (2) that social interaction as well as SWMS are important for safety; (3) gaps do exist between work as imagined in a SWMS and work as performed in practice, but in the minds of those on site, there were no unresolved gaps; (4) therefore construction companies must identify and resolve the gaps between SWMS, practice and task demands to make construction sites a safer place to work.
\end{abstract}

\section{Keywords:}

Safe work method statements; safety rules; construction safety; work as imagined; work as performed

\section{Introduction and background to the problem}

This study focuses on the role of safe work method statements (SWMS) in controlling risk in the Australian construction industry. Although construction safety has and continues to be the subject of much research effort (see for example the special edition on construction safety in the Journal of Safety Research, 2010, and other special editions on the same topic in the journals of Applied Ergonomics, 2005, and Safety Science, 2008), there is a lack of research around the role of SWMS in controlling risk. Therefore, the aim of this study was to determine how managers and workers interpret and use safe work method statements in the 
Australian construction industry in order to explore if there was a gap between work as imagined and work as performed.

\subsection{Size of the problem in Australia}

Previous studies have identified that construction work is dangerous, dynamic and exposes workers to a variety of hazards and risks, resulting in unacceptably high levels of injuries and fatalities (Aksorn \& Hadikusumo, 2008; Choudhry \& Fang, 2008; Choudhry et al., 2008; Gittleman et al., 2010; Hoonakker, et al. 2005; Loosemore \& Andonakis, 2007, McDonald et al., 2009; Mitropoulos \& Cupido, 2009; Mohamed et al., 2009; Rozenfeld et al., 2010; Suraji et al., 2001, Teo, et al. 2005; Törner \& Pousette, 2009; Zhou et al., 2008). The Australian experience is no different.

In Australia, the incidence rate of claims for injury in the construction industry has fallen by 30 per cent during the period 2000-01 to 2006-07 (Safe Work Australia, 2010). However, for the period 2007-08, the Australian construction industry still recorded an incidence rate of 21.6 serious claims per 1000 employees, making it one of four industries with an incidence rate substantially higher than the Australian all industries rate of 13.5 claims per 1000 employees (Safe Work Australia, 2010). During this same period muscular stress accounted for 34 per cent of claims, falls, trips and slips accounted for 26 per cent of claims and being hit by a moving object accounted for 16 per cent of claims (Safe Work Australia, 2010). The number of fatalities in the Australian construction industry has ranged between 45 and 53 for the period between 2000-01 and 2006-07. For the latter period, the construction industry had the fourth highest fatality incidence rate of all Australian industries, recording 52 fatalities for the period resulting in an incidence rate of 5.6 fatalities per 100,000 employees - twice that of the rate for all industries (Safe Work Australia, 2010). Long-term contact with chemicals and substances accounted for 33 per cent of fatalities, vehicle accidents accounted for 24 per cent of fatalities, falls from height accounted for 11 per cent of fatalities whilst contact with electricity accounted for 7 per cent of fatalities (Safe Work Australia, 2010).

\subsection{The National Standard for Construction Work}

In recognition of the problem, the Australian government in 2002 formally identified the construction industry as a high priority industry and directed effort toward reducing the incidence of injuries and fatalities. A Royal Commission into the building and construction industry followed this in 2003 and recommended the development of a national standard for occupational health and safety (OHS). As a result, The National Standard for Construction Work [NOHSC: 1016 (2005)] was released in 2005 with the aim of "protecting persons from the hazards associated with construction work" (NOHSC, 2005, p.1). Section six of the standard sets out the obligations to control risks in accord with a hierarchy. In the first instance, the obligation is to eliminate the risk. If elimination is not reasonably practicable, then there is a prescribed order of measures to minimise risk. SWMS statements, as an expression of a safe system of work, appear second from the bottom of the hierarchy, implying that SWMS are only an option after exhausting other measures to control risk although the standard does identify that when no single measure is sufficient, then a combination of risk control measures may be used. In practice, it is likely that a combination of risk control measures are used that includes SWMS.

The standard goes on to stipulate that "each person with control of over high-risk construction work ... gives the person with control of the construction project a written safe work method 
statement' (NOHSC, 2005, p. 20). The expectation is that construction workers will comply with the SWMS. State and Territory OHS laws tend to mirror these requirements for SWMS, in some cases referring to them as "job safety analyses" or "work method statements". For example, the Victorian Occupational Health and Safety Regulations 2007, forbid an employer from undertaking high-risk construction work unless a SWMS has been prepared in advance. Therefore, under Hale and Swuste's (1998) three types of rules: goal rules, process rules and action rules, this requirement to prepare a SWMS is a "process rule" for construction organisations and their managers, and an "action rule" for construction workers. For the latter group, a SWMS removes freedom of choice and specifies how construction workers are to behave.

Returning to the standard, the trigger for writing a SWMS is high-risk construction work. According to the standard, high-risk construction work means any one of 19 activities shown in Table 1. With the exception of muscular stress and possibly long-term contact with chemicals and substances, the common causes of injuries and fatalities in the construction industry (described previously) are accounted for in the 19 high-risk construction activities.

Table 1

High-risk construction activities

a) Construction work where there is a risk of a person falling two metres or more;

b) Construction work on telecommunications towers;

c) Construction work involving demolition;

d) Construction work involving the disturbance or removal of asbestos;

e) Construction work involving structural alterations that require temporary support to prevent collapse;

f) Construction work involving a confined space;

g) Construction work involving excavation to a depth greater than 1.5 metres;

h) The construction of tunnels;

i) Construction work involving the use of explosives;

j) Construction work on or near pressurized gas distribution mains and consumer piping;

k) Construction work on or near chemical, fuel or refrigerant lines;

l) Construction work on or near energized electrical installations and services;

m) Construction work in an area that may have a contaminated or flammable atmosphere;

n) Tilt-up and precast concrete construction work;

o) Construction work on or adjacent to roadways or railways used by road or rail traffic;

p) Work on construction sites where there is any movement of powered mobile plant;

q) Work on construction sites where there are artificial extremes of temperature;

r) Construction work in, over or adjacent to water or other liquids where there is a risk of drowning; and

s) Construction work involving diving.

Source: National Standard for Construction Work [NOHSC;1016 (2005)]

\subsection{What is a safe work method statement?}

A SWMS is defined by the Office of the Federal Safety Commissioner, a government body in Australia that audits and accredits companies seeking to tender for government building works, is a document that identifies hazards, assesses the risk of those hazards occurring and identifies the risk controls that must be put in place (OFSC, 2010). As such, a SWMS is the main document that guides the safe practices of workers on a construction site. In this regard, 
a SWMS is similar to a job safety analysis (JSA), also known as a job hazard analysis (JHA) (Rozenfeld et al., 2010). According to the US Occupational Safety and Health Administration, a JHA is defined as a "technique" for identifying hazards with a focus on the "relationship between the worker, the task, the tools, and the work environment" so as to either eliminate risk or reduce risk to an acceptable level (OHSA, 2002, p. 1). A feature of both a SWMS and a JHA is that work activities are broken down into a series of job tasks or steps and hazards and risk controls identified for each task or step. SWMS may differ from a JHA in that they require the writer to list the OHS legislation relevant to the activity. Therefore, in principle, SWMS and JHA are very similar, both sharing the purpose of identifying worker practices that will control risk at each step in an activity. In practice, the terms SWMS/JHA/JSA are sometimes used interchangeably in the Australian construction industry. However Rozenfeld et al. (2010, p. 492) argue that JSA's in their current form are "impractical for the construction industry" because of the dynamic and changing nature of construction work, although Haslam et al. (2005) argue that the idea that the construction industry is different to other industries “does not stand up to scrutiny” (p. 414). The National Standard for Construction Work requires that a person in control of a construction project must ensure that construction workers comply with the SWMS. If workers violate a SWMS, then work should stop and not resume until they comply with the SWMS. This edict of strict compliance with a safety rule at the worker level is at odds with Rozenfeld et al's (2010) view that JSA (SWMS) are impractical. Given the dynamic and changing nature of construction work, there are many opportunities for gaps to emerge between work as imagined in the SWMS and work as performed to meet the current requirements on a site. Taking the view that SWMS constitute a safety rule, then it is appropriate to ask what the evidence base tells us about safety rules in general, and in particular SWMS, in the construction industry.

\subsection{Safety rules}

Studies of the role of safety rules have occurred across a range of industries and occupational categories, but less so in the construction industry. For example, safety rules have been studied in: mining (Laurence, 2005), aviation (Dekker, 2001; Lokoupoulo, 2008; Wright \& McCarthy, 2003), seafaring (Knudsen, 2009; Nathanael \& Marmaras, 2008), manufacturing (Costella et al., 2009; Marchand et al., 1998; Simard \& Marchand, 1997), aircraft maintenance (McDonald et al., 2000), railways (Grote et al., 2009, Hale et al., 2003, HarmsRingdahl, 2004, Larsen \& Hale, 2004), wildland firefighting (Thackaberry, 2004, Weick, 1993), nuclear power plants (Dien, 1998; Bourrier, 1996;), the petroleum industry (Antonsen et al., 2008) and operating rooms (McDonald et al., 2006; McDonald et al., 2005).

Taken as a whole these studies indicate that there is a gap between practice and safety rules, which implies that writing more rules is not necessarily or in all cases the answer. Wright \& McCarthy make the point that "in practice, procedures are often adapted, modified, and circumvented on the basis of the operator's appraisal of the local situation” (p. 680). One problem with current approaches to safety rules may be the steadfast belief by managers that safety is the product of workers always complying with safety rules. Ironically, simply writing more rules may only result in more opportunities to violate the rules. However even the language of rule violation is loaded with the assumption that the rules are always right and deviant action is always wrong. This is a contestable assumption. The contrary view that emerges from much of the research around not only safety rules, but safety culture also, is that safety is an emergent property of a socio-technical system, meaning that workers seek to interpret and apply safety rules taking into account the current situation within which they find themselves, and modify or adapt the rules accordingly. In other words, they may socially 
construct what it means to work safely. This view of safety rules may be challenging for managers, safety professionals and regulators alike who may experience discomfort in letting go of the 'certainty' that is offered through prescribed safety rules, a certainty that may be more an 'illusion of safety' than real (Borys, 2009). However, the perspective that safety is socially constructed is not new, but it may be the case that is is not valued, studied and accepted in practice as much as it could be.

Rochlin (1999, p.) has previously made the point that safe operation is a social construct whilst more recently Turner and Gray (2009) have developed the same theme arguing that the "appreciation of the social construction of safety in organizations represents a distinctive shift in the mainstream approach ...” (p.1260). Failure to acknowledge that safety is also a social construction may result in a gap between procedures and practice, restricting opportunities for organisational learning (Antonsen et al. 2008, Wright \& McCarthy, 2003). Dekker (2003) argues that organisations should not place pressure on workers to comply with rules; rather managers should monitor the gap between procedures and practice and provide workers with the skills to know when and how to adapt. Alper \& Karsh (2009) support Dekker's view. In their systematic review to determine the causes of violations in industry they recommend that instead of blaming workers, managers should find out why workers violate rules and reduce the need for violations by re-designing the environment or allow workers to violate rules safely. One way to achieve the latter is through a framework for rule management, or a 'rules for rules' approach (Hale \& Borys, 2010). A managed approach to safety rules would determine not only the type and number of rules necessary, but make provisions for handling exceptions and sanctioning discretion to modify and adapt the rules under certain conditions. Such an approach gives more weight to the social construction of safety than is typically available under conventional approaches to writing safety rules.

Research relating to safety rules in the construction industry agrees that construction work is complex and dynamic, but disagrees on the role of safety rules. Some studies advocate more adaptive approaches to safety rules (Howell et al., 2002; Iszatt-White, 2007, Mitropoulos \& Cupido, 2009a Mitropoulos \& Cupido, 2009b; Mitropoulos et al., 2005; Rozenfeld et al. 2010; Saurin et al., 2008; Törner \& Pousette, 2009 ). Others advocate strict compliance with safety rules (Aksorn \& Hadikusumo, 2008; Choudhry \& Fang, 2008; Kartam et al., 2000; McDonald et al. 2009; Teo et al. 2005; Zhou et al. 2008).

Studies that focus on a more adaptive approach to safety rules could be said to be advocating a 'rules plus' strategy. This group of studies recognises the limitations of safety rules and offers other strategies to work in concert with the rules. For example, train workers to use their judgment to identify how close they are "working to the edge" of safety (Howell et al. 2002). Foster social interaction to include cooperation, mutual trust and empowerment (Törner \& Pousette, 2009). Monitor how information is exchanged between crewmembers to learn about and incorporate into pre-planning, the adaptive strategies used by crews (Saurin et al. 2008). By comparison, studies that advocate strict compliance could be said to be advocating a 'rules is rules' strategy.

Missing from research into safety rules is research into the role of SWMS in the construction industry. In Australia, a report prepared by WorkCover New South Wales in 2001 found that "documented safe work practices do not always translate to actual safe work practices. The research findings indicate that at present a 'divide' exists between paper and actual workplace compliance” (WorkCover NSW, 2001, p. 8). This quote is 
instructive for a number of reasons. Firstly, the findings in relation to SWMS are consistent with those found in the literature on safety rules. That is, there is a 'divide' or gap between work as imagined in the SWMS and work as actually performed. Secondly, two words are instructive in the above quote: "workplace compliance". Compliance with a SWMS is in keeping with the aspirations of the National Standard for Construction Work in Australia. However, it could be construed as being at odds with the literature on safety rules that leans heavily away from a strict compliance with safety rules strategy (or 'rules is rules' strategy) towards a more adaptive approach to the management of safety rules strategy (or a 'rules plus' strategy). Finally, in the nine years since this statement first appeared, no research studies have evaluated SWMS as an intervention to control risk in the construction industry. This is not surprising. A systematic review on the efficacy of interventions in the Australian construction industry, commissioned by the Office of the Federal Safety Commissioner in 2007, found that "the vast majority of technical, human factors and organisational interventions ... have not been adequately evaluated; there is an urgent need to address this gap in the evidence base” (OFSC, 2007, p. 2). The same applies to SWMS, which has been a little-researched area. Therefore, this study attempted to answer the following research questions:

1. How do managers interpret and expect safe work method statements to be used?

2. How do workers interpret safe work method statements and use them in practice?

3. Is there a gap between the safe work method statements and workers' safety practices?

4. Do workers adapt their safety practices to suit local circumstances?

5. What factors drive the process of adaptation?

\section{Methodology}

\subsection{Study Design}

This study used symbolical interactionism as a theoretical perspective for studying how managers and workers interpret and use SWMS on a construction site. This perspective informed the development of the interview questions and the researcher's stance towards interpreting the results. Such a perspective is consistent with the view that safety is socially constructed. For construction workers, to follow, or to modify and adapt safety rules (including SWMS), is an interpretive process embodied within Weick’s (1995) concept of sensemaking. According to Weick, sensemaking is akin to symbolic interactionism because both concepts acknowledge the importance of "self, action, interaction, interpretation, meaning and joint action” (p.41). Charon (2010), in summarising symbolic interactionism as a sociological perspective, states that: "to understand human action, we must focus on social interaction, human thinking, definition of the situation, the present, and the active nature of human being” (p.29).

The theory of symbolic interactionism rests on three premises (Blumer, 1969). Firstly, "human beings act towards things on the basis of the meanings that the things have for them" (p. 2). Construction workers, different groups of workers, supervisors and managers may act differently toward SWMS based upon the meanings that both groups attach to these documents. The second premise is that the "meaning of such things is derived from and arises out of, the social interaction that one has with one's fellows" (p.2). Therefore, how workers interact with one another and with their supervisors and managers around SWMS may 
influence the meanings that different groups hold on the one construction site in relation to SWMS. The third premise of symbolic interactionism is that "meanings are handled in, and modified through, an interpretative process used by the person dealing with the things he [sic] encounters" (p. 2). This could mean that construction workers may modify what SWMS mean to them in controlling risk based on how they interpret work as it unfolds around them.

Social interaction is the genesis, over time, of culture (Charon, 2010). SWMS are a form of social object that, if afforded meaning, will be used as a symbol to stand for safety and will be communicated as such within the culture (Charon, 2010). The reverse is also true. Afforded a different meaning, SWMS may be a symbol that stands not for safety, but for bureaucracy and an impediment to getting the job done safely. This study used ethnography as a methodology as it is an accepted approach for studying culture (Fetterman, 1989; Geertz, 1975).

\subsection{Setting and Participants}

This study was conducted in two construction projects under the control of the one multinational construction company in Australia. The projects were chosen using Neuman's (2003) three criteria for choosing a site for field research: richness of data, unfamiliarity and suitability. Both projects were a rich source of data as both made extensive use of SWMS, the two construction projects were unfamiliar to the researcher and finally the organisation, as well as each individual project agreed to participate in the study.

Project one was as a 24-level hotel complex being built on a narrow footprint and located in the central business district of a large metropolitan city in Australia. This project was nearing completion and was undergoing 'finishes and defects'. Project two, located in the same Australian city, was a 22-level hotel complex being built on a small corner block. The small corner footprint meant that the tower crane was set up on the road way and pedestrians had to be routed around the project at street level. Construction had only reached the 13th floor and a tower crane and fixed hoist were both operating on site.

The focus of this study was restricted to employees of the principal contractor engaged in a range of activities spanning both projects including activities deemed to be high-risk construction activities, for example, work where there is a risk of a person falling two metres or more and movement of powered mobile plant. A purposive sample (Neuman, 2003) of project managers, site managers, supervisors and labourers were recruited from across the two projects to gain different perspectives on the role of SWMS. A purposive sample is selected using predefined criteria (DePoy \& Gitlin, 2005). The key criterion used by this study was that participants had or were currently required to develop and/or use SWMS. Access to the participants was organised through the site manager for project one and the project manager for project two. Prior to this, the researcher spent six months 'getting to know' the organisation. The researcher met with each participant inviting them to participate in the study. Access to some participants was restricted due to workload pressures, limiting the number who could participate. The 18 participants had an average length of service with the construction company of 8 years and an average length of service in the construction industry of 18 years. Ten were construction labourers, four were supervisors, two were site managers and two were projects managers. All participants were male.

Approval for the study was obtained from the University of Ballarat Human Research and Ethics Committee. All participants were provided with a Plain Language Information Statement and provided signed consent 


\subsection{Data collection}

Three methods of data collection consistent with an ethnographic approach were used: semistructured face-to-face interviews, a review of SWMS and a site walk. Semi-structured interviews addressed the purpose of SWMS, the importance of SWMS, how SWMS are developed and monitored, the extent to which SWMS were applicable to the reality of work on site, what workers rely on to get the job done and maintain a safe workplace and the extent to which SWMS influence safety on a construction site. All interviews were digitally recorded.

A schedule of planned non-participant observations by the researcher did not go ahead as the organisation elected not to grant approval for this method of data collection. This resulted in what Antonsen (2009) refers to as an ethnographically inspired approach, where the basic stance towards the participants is as important as the time that can be spent observing. This is in contrast to an orthodox ethnography which normally entails sustained periods of being immersed in the culture under study.

\subsection{Data analysis}

Analysis of qualitative data sacrifices what Silverman (2010, p. 104) refers to as "scope for detail”. Detail in the context of this study refers to how workers and managers interpret and use SWMS and the subsequent meaning they attach to these documents. Detail also refers to how workers and managers deal with any gaps between SWMS and practice. This detail is available to the researcher through the words workers and managers use to discuss their experiences with SWMS during interview. There were three stages to the data analysis. Stage one involved transcribing the recorded interviews and entering the transcriptions into NVivo 8 software ready for further analysis. However, qualitative data analysis is not a discrete process and permeates all stages of the research including data collection, data transcription as well as the more 'formal' analysis stage (Silverman, 2010). Stage two involved close reading and open coding (Emerson et al., 1995) of the transcribed data. Three types of codes were used: descriptive codes that entail little interpretation, interpretive codes and pattern codes that identify an emerging theme (Miles \& Huberman, 1994). These codes were attached to words, sentences and whole paragraphs. An example of each type of code follows:

1. Descriptive code: SWMS are 'absolutely applicable' (voice of the workplace).

2. Interpretive code: The continuing reference to talking and communication was coded as 'safety is about planning and communication' (voice of the researcher).

3. Pattern code: The recurring reference to tasks that were 'out of the ordinary' (voice of the workplace).

Stage three used Miles \& Huberman's (1994) principle of data reduction to organise codes into fours themes related to how managers and workers interpret and use SWMS. Focused coding (Emerson et al., 1995) was undertaken within each of the four themes to look for common and dissenting views, patterns and ways of making an argument or telling a story within and across each occupational category. For example, by aggregating the code, 'absolutely applicable' along with similar codes, led to the theme 'SWMS are important for safety'. This theme, along with the other three themes discussed in the next section, allows a storyline to be developed that will address the research questions. 


\section{Results}

The results of this study are presented according to four themes that emerged from the data: i) SWMS are important for safety, ii) Reserve SWMS for tasks that are out of the ordinary, iii) social interaction as well as SWMS is important for safety and iv) no unresolved gaps between SWMS and practice. For each theme, descriptive results for the four occupational groups interviewed are presented first, followed by a brief analysis of the data within each group and between the groups. In presenting the results, the terms SWMS and JSA mean the same thing.

\subsection{SWMS are important for safety}

This theme explores the idea that all four groups: labourers, supervisors, site managers and project managers interpret SWMS to be important for safety, albeit for subtly different reasons, but a challenge emerges regarding the use and coverage of SWMS in practice.

Labourers deemed SWMS important for safety for personal safety and risk control reasons. For example, one labourer said the following in relation to personal safety:

All JSAs are important ... it is important for those people involved in that JSA in terms of their own safety.

They deemed SWMS particularly important for high-risk jobs, young workers, special projects, important jobs and for infrequent jobs. Generally, labourers had a positive view of SWMS, with one labourer going so far as to say 'the JSA is the Bible of how you do things'. For others, even though they viewed the requirement to follow them as extreme, they saw them as a form of procedure that if not followed, would increase risk, as these labourers said:

Sometimes it's very extreme as in strict, but sometimes it has to be that way. There is no other way. It's safety. It's number one.

The JSA is important in that respect in ensuring that nobody else is endangered. Without following that procedure there are obvious risks.

Sometimes, however, the SWMS would be adapted to meet the changing circumstances on site. However, there was a view that you do not need a SWMS for everything, and labourers sometimes valued their experience and competence as much as the SWMS as the following quotes illustrate:

What I have found over the past couple of years is there is a JSA for everything. I don't think that is necessary.

Sometimes you have JSAs for minor jobs and they can be unnecessary. An everyday lift type of thing someone might decide we need to do a JSA on it and it is totally unnecessary. It is something you do all the time and the blokes who are involved know exactly what has to be done. It is not necessary to have a JSA in the first place. But for one offs I think it is important.

Supervisors deemed SWMS important for safety, particularly for high-risk activities or major tasks, because they did not want any accidents. They attributed a decrease in injuries in the construction industry over the last decade and a half to the introduction of SWMS. They also felt that SWMS helped mitigate their personal liability in the case of an accident. However, 
supervisors felt that SWMS increased the amount of paperwork that they had to deal with which took their time away from performing other tasks, as the following quotes illustrate:

For high-risk activities, they are very important, but there is one thing with them they are more paperwork and it is impossible for supervisors to do all the paperwork they are supposed to do.

I suppose they are a necessary evil. They are good in one way, you get a chance to sit down and go through the process with the boys. They take a lot of time normally, normally when you are under the pump you will find there isn't a JSA already written out for a particular item ... sometimes they are a right royal pain in the bum, but we have to do them I suppose for all the major tasks.

The quotes above also suggest that because SWMS impose time constraints upon supervisors, their relationship to SWMS is more ambiguous and this ambiguity is captured in the sentiment that SWMS are a "necessary evil" and a "right royal pain in the bum".

Site managers deemed SWMS important for safety for financial, moral and risk control reasons. They too felt that SWMS helped them to mitigate personal risk. However, site managers believed that there were too many SWMS. As a result, the important ones do not 'stick out'. One solution offered was to value competence to control low risk tasks and a SWMS combined with competence to control high-risk tasks. The following series of quotes illustrates this point:

One thing I probably don't agree with so much, although it mitigates my element of risk, is that you get an influx of a lot of generic JSAs. These are really activities that you wouldn't consider as a real risk. It's just more telling you for the sake of telling you, or having a JSA for the sake of having a JSA, which I don't believe in. Once you go and flog the guys with 100 JSAs and induction when they walk on the job, the more important ones don't tend to stick out from a lot of the ones which ... it is just taking the impact out of the more serious JSAs.

This site manager goes on to provide an example to support his point:

Things like having a JSA on how someone needs know how to fix a skirting to a wall for example. You might have this carpenter who is fixing a skirting on the wall, but in the same breath he is out doing some sort of façade treatment on the outside of a building, which is significantly high risk to something which I see as a lower risk. He has gone through 15 JSAs and within that, you have got a high risk one and one that is probably more skilled based if he is a tradesman as well and probably only needs a company induction as opposed to a JSA. So what I am saying you put the guy in a room for an hour and a half and you induct him with all these JSAs. He has covered things that have gone from one extreme to another. So what is the impact of the real high risk activity if you are leave that to the last? He is going be in la-la land by the time he gets to that.

To overcome this problem, the site manager proposed the following:

The way I see it, if you have a particular person who has a competency in working as a tradesman, say, for example a carpenter, one who has gone through an apprenticeship, he is qualified. He obviously knows how to use a hammer and install a skirting. So where its something where its trained in a competency as being a tradesmen then I think it only needs to be covered in company induction or expectations of induction or something like that ... because to tell a carpenter how to swing a hammer is probably not something that I think needs to be included in a JSA. But to tell a carpenter how to swing a hammer when he is hanging of the edge off a building, that is something that needs to be included in a JSA ...

These remarks by the site manager suggest that workers warrant a degree of discretionary space based upon their competence and the context within which they are working, which may not be codified in rules. Implicit in this statement by the site manager is that writing rules for all tasks including generic SWMS may not only interfere with getting the job done, but 
may also devalue the skill, judgment and ultimately identity of qualified tradespeople. This statement by the site manager does not explicitly acknowledge a gap between SWMS and practice, but nor does it rule it out. What is explicit, however, is an issue of rule coverage and workers, supervisors and managers alike are questioning the need to write a rule for all tasks. Writing rules, in this case SWMS, in particular generic SWMS, for all tasks runs the risk of workers ignoring rules for lower risk tasks and preferring instead to rely upon their competency as a more suitable means of risk control.

Finally, project managers, similar to site managers, deemed SWMS important for safety for risk control reasons, but also thought that SWMS were a useful planning tool. Project managers believe that SWMS trigger workers to think about risk on the job. Implicit in this last comment is the assumption that SWMS, thinking about risk and work practices that comply with the SWMS are related. Ironically, by extending SWMS to cover all taks, the less valid this assumption may become.

Overall, all groups deemed SWMS important for safety but for slightly different reasons. For some, but not all workers, safety and the role of SWMS as a means to control risk was personalised, most likely because they are the ones who will suffer the consequences if something goes wrong. Supervisors and site managers also personalised safety and the role of SWMS, but as one way to protect them from litigation in the event of an accident. In addition, supervisors seemed to be under time pressure and wear the day-to-day burden of developing, monitoring and enforcing the use of SWMS on top of their other duties. By comparison, project managers had a less personalised view of safety and the role of SWMS, but clearly articulated a view of how SWMS influence safety through planning, risk control and encouraging labourers to think about risk. All groups, with the exception of project managers, deemed SWMS important for safety, but only up to a point, after which, there was consensus that too many SWMS were not beneficial and in fact quite the reverse. That is, there is a risk that the essential few that relate to critical risks many not stand out from the trivial many. Some labourers and site managers shared the view that competence, as well as SWMS, had a role to play in controlling risk.

\subsection{Reserve SWMS for tasks that are out of the ordinary}

This theme develops the issue of rule coverage introduced in the previous theme with support emerging from among the groups that SWMS should be reserved for tasks that are out of the ordinary.

Labourers deemed SWMS important for work that was out of the ordinary as opposed to 'every little thing', in particular, high-risk work. For example, one labourer made the point:

You are not going to do a JSA for every little thing, if its high-risk work then you will do the JSA.

High-risk work for labourers included crane work, work involving the use of machinery, work that may affect pedestrians and work that may encroach upon roads. Once identified as being 'out of the ordinary', planning for that job, including the preparation of a SWMS, was viewed as being vitally important so that everyone understood their role, how the job will take place, the hazards and risks involved, and the ways to control them. The concept of 'out of the ordinary' also extended to novices on site who would be unfamiliar with the hazards and risks and ways to avoid or control them. Furthermore, out of the ordinary extended to dealing with exceptions. In this case, the SWMS may have to be adapted to meet the dynamic nature of the job. This starts to suggest that under certain circumstances, there may be a gap between 
SWMS and the practices need to complete the job safely. Any ensuing gap would be resolved through consultation between labourers, supervisors and shop stewards leading to a revision of the SWMS. The following quote highlights how they deal with exceptions:

Sometimes if the job is dynamic sometimes we may need to alter a JSA and that happens in conjunction with the foremen and the relevant shop stewards and we may offer suggestions, however the written work on the actual changing of the plan is done by management.

This labourer goes on to provide an example of a dynamic job:

Working in conjunction with overhead cranes and people working below and on split levels. Where you have several contractors working in proximity and it makes the job even more so, makes the JSA even more so a precautionary procedure because of the dangers involved, the multiple dangers in the area at any one given time.

Supervisors also deemed SWMS important for work that was out of the ordinary and not for 'every little thing', in particular, high-risk work. For example:

They are very important. If you were to write a JSA for every small thing that would be too much. But for high risk tasks you definitely need to get everyone together so that everyone understands what is going to happen. Normally when we do anything out of the ordinary we would have a toolbox before it, go though it, so that everyone would know what is happening and then hopefully everyone would work the same way.

Supervisors saw themselves as playing a vital role in the planning process for high-risk work, including the preparation and communication of SWMS, as they are in a position to know what work is coming up. When exceptional circumstances arise, supervisors would decide on an alternative method of performing the work safely. Because of time constraints, supervisors may decide to 'press on' with the work rather than spend time revising the SWMS, exceptions would, however, be used as an opportunity to learn and the lessons learnt would be included in future SWMS. This is a further example of where a gap exists between SWMS and particular task demands, as one supervisor explains:

We are going to do a job; it might be removing screens, formwork screens - one of our contractors removing screens. He has a JSA to remove screens and his JSA spells out how he is going to do that. Then you get something that wasn't standard and you end up with someone out in the middle of nowhere and no one has got wings you can't fly out there. So 'OK what are we going to do'? If you can't get it off the scissor lift and 'no', you can't put a harness on and walk out on the needle, we are going to have to stop, put the chains down, pick up the crane box, get out there in the crane box do it this way. They have done a JSA for the crane box, it's the safest way to go. What you should do is probably stop and go back to the office and re-write a JSA and two hours later come back and finish the task, but that ain't going to happen. You need to press on and finish what you are doing but remember that for next time.

The quote above suggests that they close the gap by altering their practices even though a gap between the SWMS and the task demand may remain. The opposing view was that accidents occur when circumstances change and the SWMS is not revised to reflect those changes.

Site managers too deemed SWMS important for work that was out of the ordinary and out of the ordinary work is synonymous with high-risk work. High-risk work for site managers included pulling the crane down. For site managers, planning is the key for managing highrisk work, at times piloting the approach to the tasks and revising the SWMS accordingly. Project managers did not necessarily look at every SWMS, but reviewed SWMS for high-risk or out of the ordinary tasks, for example removing a crane. Project managers would monitor 
these high-risk activities against the SWMS as the job proceeded and follow-up any deviations from the SWMS with supervisors.

There was agreement among the groups that SWMS were most appropriate for tasks that were out of the ordinary, and by definition, 'not for every little thing'. Out of the ordinary was synonymous with high-risk tasks. The other common feature across the groups was that planning for high risk-tasks was an essential feature of practice on site. Other 'out of the ordinary' activities were exceptions, for example, changing weather conditions. Exceptions were also more likely with high-risk tasks. Labourers dealt with exceptions through a process of consultation resulting in a revised SWMS. Some supervisors were more liberal with how they dealt with exceptions. Because supervisors experience time pressure, some may decide on a safer way to get the job done and update the SWMS later. Exceptions were not mentioned by either site or project managers. They took a much more 'hands on' approach to developing, monitoring and enforcing SWMS. In one case, exceptions were dealt with through a planning process where in effect, exceptions were identified and resolved through a trial run through of the approach to the task.

This theme has developed the idea of rule coverage as an issue but has also started to reveal that gaps are the norm between SWMS and tasks that are out of the ordinary. These gaps are resolved through a process of either formal or informal consultation - a topic developed in the next theme. It is unclear from this study if similar gaps exist for ordinary tasks, although it is likely that there will always be gaps with greater rule coverage possibly inciting more gaps. Therefore, monitoring for and resolving a gap for all types of work becomes important work as part of the safety management system. The next section further develops the importance of consultation within the context of social interaction or the broader social milieu.

\subsection{Social interaction as well as SWMS is important for safety}

The previous theme discussed how consultation was essential for resolving gaps between SWMS and work that is out of the ordinary. This theme amplifies the importance of consultation and places it in the broader context of social interaction as well as SWMS as being important for safety.

For labourers, social interaction took place around the writing of SWMS and in relation to working safely on site. Most labourers said they had little or no input into the writing of SWMS, for example, one labourer said:

I wouldn't have any. Well you would put forward suggestions if you like ...usually when it comes to us the JSA is already written up on how to approach something, they would run it past a gang of men ... how they are going to do the job we could make suggestions at that stage to change things. I suppose for what we would be doing I suppose it would come from the office ... but that would be normally written up by the time we get to where I'm at and you just follow those guidelines.

From their perspective 'management' or the 'foreman' wrote SWMS. One labourer said that it was 'not their job' to write SWMS. Labourers did say, however, that even though 'by the time it comes to us it is written out', supervisor do ask them to 'put forward suggestions'. The company uses toolbox meetings to induct labourers into the written SWMS. Toolbox meetings provide a further opportunity for labourers to have input into the SWMS, or as one worker said: 
Well that's where it's good when we have a toolbox ... they might have missed something. We can speak up and then we put it in the JSA.

Labourers were of the view that working safely on site comes about through a process of talking to one another, talking to 'subbies' (sub-contractors) and talking to their supervisor. At times talking to one another, or as one labourer expressed it 'have a whisper', was one strategy that they use to encourage one another to follow the SWMS or to work safely. In other cases, having a 'bit of a talk' is important to gain respect so that different groups on site are aware of what the other is doing. Labourers may also initiate interaction with their supervisor if they are 'not comfortable' doing a job or a supervisor may pull labourers up if they get a 'bit slack'.

Supervisors concur that they write 90 per cent of the SWMS for their own labourers, but that they do so in consultation, as one supervisor said:

90 per cent if it was our own labourers I would actually be doing the writing myself but while talking to them, so it is their words but I am putting it in writing just because most of them wouldn't have access to the computers.

Some supervisors said that consulting labourers on the best way to do the task builds trust. Supervisors also interact with labourers in relation to violations of SWMS. However, some supervisors prefer to informally deal with violations with the offending labourer. For example, one supervisor said:

You just let them know that they are doing it wrong. I know a guy the other day was standing on top of the scissor and I just said, "that's not how you use the scissor" and he knew straight away, you don't stand on the top.

From the perspective of the site manager, labourers are involved in writing SWMS, particularly for high-risk tasks.

Social interaction takes place formally and informally and both are important for the social control of risk. Formal interaction takes place around the writing of SWMS before a job commences. Informal interaction takes place out on site whilst work is being performed between labourers and between labourers and supervisors. Labourers keep an eye-out for the safety of one-another and for 'subbies', being prepared to intervene if they observe someone doing something that could put them or others at risk. Supervisors interact with labourers in relation to monitoring compliance with SWMS and counselling labourers for violations.

This theme adds a new dimension to the role of SWMS by locating these documents in the broader social milieu or culture that is instrumental for working safely on site. This social milieu or culture for safety, achieved through social interaction may operate as a 'work around' to what is perceived as the onerous rule coverage associated with SWMS.

\subsection{No unresolved gaps between SWMS and practice}

There are a number of and sometimes-competing discourses around safety rules, with one discourse surrounding the notion of a gap between work as imagined and work as performed. In introducing this theme, it is worth keeping in mind in the context of this study that the discourse of no unresolved gaps is not necessarily the same as saying there are no gaps. The other themes have suggested such gaps do exist. This discourse of no unresolved gaps is one 
that is embedded in the language of compliance and starts to raise the question: which gap are we interested in?

Labourers did not acknowledge a gap between SWMS and practice. The following comments typify this 'no un resolved gap' view, a view sometimes based on the premise that labourers trust the experience of supervisors and managers to write SWMS that are relevant to their work:

Not really, most of the time the guys in the office have been around and they know what is going on, but most of the time they seem they're suited to what we've got to do.

Well most of the JSAs are pretty good, the ones that [name of supervisor] normally does, he has been in the building trade for a long time and is pretty switched on, he knows what we can cope with, what we can do and what we can't do he knows that ... normally I think they are pretty good.

I think every time we have done the JSA it has been relevant ... so I can't think of a time where I have done a JSA or where I thought this is ridiculous.

Any time we have done one I suppose it was suitable. Whether I thought it was necessary or not I don't know. I suppose they are always suitable because they let the people know what is going on and how it is going to be done, again I would go back to the same thing and ask 'is it necessary?'

Supervisors said that compliance with SWMS was high, for example, one supervisor said:

On this particular job I would say probably 95 per cent. On a bigger job like at the [mentions name of previous building project] last year that would probably drop a bit on bigger jobs just because of the fact of so much area, so big, so many people ... that could change pretty quickly and would probably drop down to $85 \%$ for the bigger jobs.

Site managers also said that compliance with SWMS was high, particularly for high-risk activities. If the SWMS does not match what labourers are required to do, or if labourers practices deviate from the SWMS, then there is a mechanism on site to deal with these situations as the following quote illustrates:

For high-risk activities I would say if you want a percentage, I think it would be up around the 9095\%, I would say for high risk activities it would be that high. If the JSA doesn't match what they are doing then we have a mechanism in place where there is enough people to keep an eye on it both from the staff and from a union OH\&S issue. If people start to vary from what their JSA says, then normally it gets picked up by one of the parties and as I say the safety net is always the Union as well.

Similarly, project managers agree that there was high-compliance with SWMS, as evidenced by what they said:

Again, I think nine times out of 10 they do, it is very important for us that they do and I would like to think that they do the whole time. I don't knowingly know of jobs we are doing out there are not matching our SWMS, if I did I would stop it.

I know this is in regards to what is on paper versus what actually happens on site and I think SWMS don't fall into that trap of being a waste of time, I honestly think they do follow them and they know how important they are.

Labelling this theme as 'no unresolved gaps' draws on the previous three themes where the language of the workplace, however implicit, provides evidence that there are gaps between SWMS and practice, or at the very least is not possible to rule out that there are no gaps. The language of the workplace associated with this theme is however somewhat more harsh and 
tends to support a discourse on safety rules most often associated with the language of rule compliance and its antithesis, rule violation. Missing are the softer edges of the social milieu, to be replaced with a more rational mechanistic view of what it means to work safely.

However, even the language of compliance is revealing in so much that compliance is never $100 \%$, indicating that gaps must exist. The language of no un resolved gaps may still be closer to reality rather than the rhetoric of compliance. The discourse of compliance and violations creates its own tensions, possibly obscuring, or even worse, resulting in denial of any gap.

\section{Discussion}

The literature on safety rules divides itself around two discourses. First, reasons for rule violations and the subsequent view that rules violations are always bad - a discourse that strives for better compliance with safety rules. Second, there will always be a gap between work as imagined and work as done, and the challenge is to make visible and resolve such gaps. This research emerges from and contributes to the second discourse. Few studies have attempted to explore the role of SWMS as a form of safety rule widely used in the Australian construction industry. This research is a first step toward filling this void by exploring how managers and workers interpret and use SWMS, and if there is a gap between SWMS and practice. The results suggest that both workers and managers believe that SWMS are important for safety, albeit for different reasons. They agree, however, that SWMS should not cover all tasks on a construction site and be restricted to tasks that are out of the ordinary. This is an issue of rule coverage and supports anecdotal impressions within the Australian construction industry that not only are there too many SWMS, but also the impression that individual SWMS are too long and detailed.

A further important finding is that there was no unresolved gap between SWMS and practice, but the key word here is 'unresolved'. Saying there is no unresolved gap is not the same as saying that there is no gap. In fact, this study did uncover gaps between SWMS and particular work tasks, in particular those deemed out the ordinary. However, the nature of any gap may be temporary as it is rapidly resolved. This study cannot rule out that there are no gaps, a finding consistent with the literature, although the finding that there were no unresolved gaps may at first appearance be counter intuitive when compared with the same literature. In choosing the label 'no un resolved gaps' the perceptions and language of those at the workplace under study is respected, where the notion of a gap did not appear to be an issue. That is, it is not so much a case of there being no gaps, but that there were no un resolved gaps because those on site had become adept at closing gaps through a combination formal and informal processes of social interaction.

However, this study does raise the question, which gap are we interested in? Three possible answers to this question emerge from this study. First, the gap between SWMS (safety rules) and practice, or what might be termed a 'compliance gap'. The language of compliance with SWMS emerged in this study, particularly among managers who may be interested in such matters, not only for risk control, but also for reasons of personal liability. This gap is associated with the content of the SWMS that is, the specific safe behaviours required of workers on site. Second, the gap between SWMS and the reality of task demands, or what might be termed an 'adequacy gap'. This is associated with the issue of rule coverage that emerged, as common ground among all four groups with the common cry of; we do not need SWMS for "every little thing". However, this gap also raises the issue of how well SWMS 
reflect the reality of task demands. In Australia, the use of generic SWMS may undermine their adequacy. Third, the gap between practice and the reality of task demands, or what might be characterised as a “competence gap'. Again, support for valuing competence over paperwork emerged as a concern, surprisingly from a site manager. Figure 1 illustrates the relationship between the gaps; gaps that are resolved through an on going process of social interaction and rule adaptation as the norm depending on the reality of task demands as their reference point. These social interactions and attendant practices are the genesis (Charon, 2010) of a culture of safety that attaches a particular and positive meaning to the role of SWMS and may result in safer construction work. Conceptualising the gaps in this way allows an organisation to question the mix of competence and rules required to meet specific task demands and perform a task safely. For example, practices compliant with inadequate rules are unlikely to meet the task demands and may lead to a decrease in safety.

\section{Task demands}

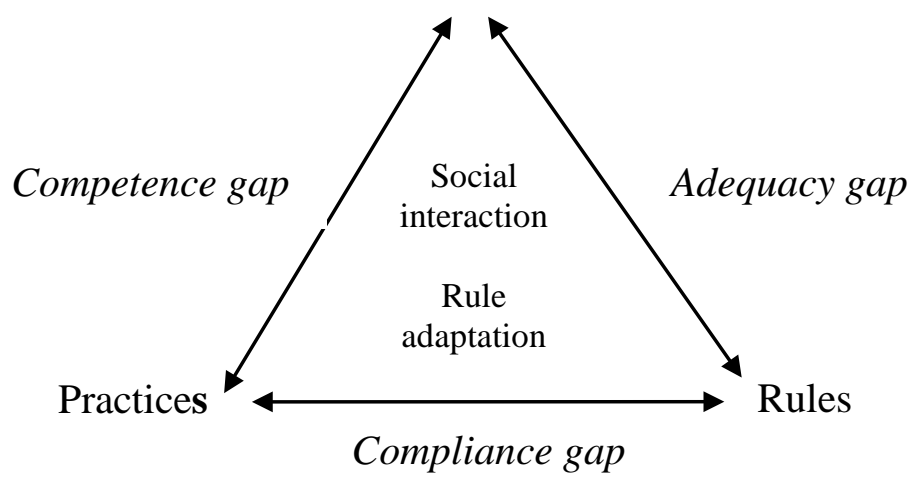

Figure 1. Three gaps of interest for working safely

The remainder of this discussion will explore explanations for the no unresolved gaps finding in this study, and with the three gaps for working safely in mind, offer potential ways forward.

\subsection{Rules about rules}

A potential explanation for the 'no unresolved gap' finding could be that workers, supervisors and managers have the ability to adapt SWMS to meet local situations either before a job through tool box meetings or on the job, giving them a sense of control which results in the perception that there is 'no unresolved gap'. By definition, the need to adapt suggests that on occasion, there is a gap, but in the minds of workers and supervisors, there is not, in other words, their sense of control closes the gap. This sense of control allows workers and supervisors to define SWMS as a symbol that is important for safety, at least for high-risk or out of the ordinary activities. As a symbol that has come to represent safety, SWMS help workers to achieve their goal of working safely, and supervisors and managers to achieve their goal of controlling risk on the job. Supervisors are a particularly vulnerable group. They are required to juggle multiple goals in a finite amount of time. Having the room to adapt SWMS to meet local situations may provide them with some breathing space in an otherwise hectic day. Particularly if they do not spend time updating the SWMS at the time, and instead get on with doing the job. Perversely, however, adapting SWMS in this manner may be at odds with their goal of mitigating personal liability in the case of an accident. This sense of 
control and the ability to adapt SWMS may explain why there is good "compliance" with SWMS. The organisation did not have a formal process for adapting SWMS, leaving them more vulnerable to prosecution in the event of an accident. Similar to initiatives in the nuclear power and rail industry (Bourrier, 1996, Hale et al., 2003) construction companies may benefit from having a formal process that addresses who can adapt SWMS, and under what circumstances, in other words, rules about rules. The framework in Figure 1 may be a useful foundation for building such a process.

\subsection{Talking rules}

Involving workers in the writing of safety rules is seen as one strategy for achieving better compliance (Antonsen, 2009). This study found, however, that supervisors and managers write SWMS. Workers have little or no input into the writing of them, in fact, they do not see it as their job, yet there was good "compliance" with the SWMS. This finding suggests two things. Firstly, workers need to have a voice and for their suggestions to be incorporated into the SWMS. That way, workers continue to perceive SWMS as meaningful and as a symbol of safety that will help them achieve their personal goal of working safely, resulting in good compliance. In this case, writing is less important for compliance than having a voice. Having a voice results in a SWMS better tuned to reality, one that has a better chance of compliance. Secondly, workers trusted the expertise and experience of their supervisors to write SWMS. Therefore, when workers are not involved in writing SWMS, compliance may be contingent upon them still having a voice and trusting who is doing the writing. On the job, compliance takes on a more subtle meaning with a move away from the language of compliance to achieving the broader goal of working safely. This is achieved through informal social interaction between worker and worker exemplified in the comment "have a whisper" and the reciprocal interaction between worker and supervisor. The findings indicate that supervisors prefer to use informal processes to counsel workers who are not fulfilling the goal of working safely.

Taken together, these processes of social interaction underline the importance of culture and the social control of risk as an ongoing process that operates in and around the more formal machinations of safety rules and OHS management systems. The implications for the construction industry, and possibly for the use of safety rules in other industries, is to talk more about the goal of working safely rather than the goal of rule compliance - even though working safely may include the need to comply with safety rules. For workers, the latter may not mean the same thing as the former. If leadership is the management of meaning, and in leading, they enact “a particular form of social reality” (Smircich \& Morgan, 1982, p.257) then there may be wisdom in leaders thinking carefully about how they frame (Bean \& Hamilton, 2006) their safety messages. Framing safety as "working safely” enacts a different social reality in the minds of workers to "working to rule" and workers may respond more positively to the working safely message than working to rule because it is more consistent with their personal goals, in this instance, talking rules.

\subsection{The critical few}

This study found that there was a perception that there were too many SWMS and that they should be reserved for high-risk or out of the ordinary activities - a finding consistent with the intent of the National Standard for Construction Work in Australian and regulations. The findings suggest that having too many SWMS may result in the critical few for high-risk or out of the ordinary activities being lost in a sea of the trivial many. Studies have found that 
having too many rules has been detrimental to working safely (Grote et. al, 2009). A potential explanation for the more SWMS is better strategy is that supervisors and managers fear personal liability in the event that something should go wrong. This is evident from the finding that one goal for supervisors and managers is to mitigate their personal liability. This creates a tension for this group as they attempt to balance their desire for fewer SWMS whilst writing more in the hope of protecting themselves legally. This is an example of where the one social object, a SWMS, represents a dual and conflicted meaning. Whilst SWMS are a social object that is a symbol for safety for high-risk and out of the ordinary activities, they are also a social object that is a symbol of protection. In the latter case, however, SWMS for lower risk and routine tasks may interrupt their other goals of achieving risk control as a building rises. Further, the findings suggest that competence and experience are the preferred mechanism for risk control for lower risk and routine tasks. One way around this dilemma is a framework for rule management that articulates what type of rules, for example, goal, process and action rules (Hale \& Swuste, 1998) and for what type of activities and risks. This would require a high degree of trust, not only between supervisors, managers and workers, but also between regulators, organisations and their managers. An interesting possibility is that whilst the sense of control closes the gap for high-risk and out of the ordinary tasks, the real gap may be for lower risk and routine tasks. This may be because SWMS are defined in the negative for these tasks and control has been taken away from workers, supervisors and managers and is applied by the external hand of the regulator. In short, lack of control may widen the gap and this is an argument for identifying the critical few.

\subsection{Limitations of the study}

This study of SWMS in the Australian construction industry has a number of significant limitations. The first is that the researcher was unable to undertake site observations and consequently the data suffers from a lack of triangulation between observations as planned, interviews and copies of SWMS. Instead, the study has had to rely on interview data only from a small sample (18 participants) across two sites of the one construction company. Undertaking observations on site to better understand the social processes for risk control is an area for further study. This study was also restricted to employees of the principal contractor and it is possible that the results would be different if it included other actors in the system surrounding the use of SWMS including sub-contractors, regulators, auditors, industry trainers and unions. This represents an important area for further study. However, as an initial exploratory study it does shed new light on the role of SWMS in the Australian construction industry, which up until this point in time, are a largely taken-for-granted and heavily relied upon strategy for risk control.

\section{Conclusions}

The aim of this study was to determine how managers and workers interpret and use safe work method statements in the Australian construction industry in order to explore if there was a gap between work as imagined and work as performed. It is one of few, if any studies to focus on the role of SWMS. This study found: (1) that SWMS are important for safety, particularly for tasks that are out of the ordinary; (2) that social interaction as well as SWMS are important for safety; (3) gaps do exist between work as imagined in a SWMS and work as performed in practice, but in the minds of those on site, there were no unresolved gaps; (4) therefore construction companies must identify and resolve the gaps between SWMS, practice and task demands to make construction sites a safer place to work. 


\section{References}

Aksorn, T. \& Hadikusumo, B.H.W., 2008. Critical success factors influencing safety program performance in Thai construction projects. Safety Science 46, 709-727.

Alper, S.J. \& Karsh, B-T., 2009. A systematic review of safety violations in industry. Accident Analysis and Prevention 41, 739-754.

Antonsen, S., 2009. Safety Culture: Theory, Method and Improvement. Ashgate, Surrey, England.

Antonsen, S., Almklov, P. \& Fenstad, J., 2008. Reducing the gap between procedures and practice - Lessons from a successful safety intervention. Safety Science Monitor 12(1): Article 2.

Bean, C.J. \& Hamilton, F.E., 2006. Leader framing and follower sensemaking: Response to downsizing in the brave new world. Human Relations 59(3), 321-349.

Blumer, H., 1969. Symbolic Interactionism. E Prentice-Hall, Edgewood Cliffs, N.J.

Borys, D., 2009. Exploring risk-awareness as a cultural approach to safety: Exposing the gap between work as imagined and work as actually performed. Safety Science Monitor 13(2), Article 3.

Bourrier, M., 1996. Organizing maintenance work at two American nuclear power plants. Journal of Contingencies and Crisis Management 4(2), 104-112.

Charon, J.M., 2010. Symbolic Interactionism: An Introduction, An Interpretation, An Integration $\left(10^{\text {th }}\right.$ Ed.) Pearson, Boston, MA.

Choudhry, R.M. \& Fang, D., 2008. Why operatives engage in unsafe work behavior: Investigating factors on construction sites. Safety Science 46, 566-584.

Choudhry, R.M, Fang, D. \& Ahmed, S.M., 2008. Safety management in construction. Journal of Professional Issues in Engineering and Practice 134(1), 20-31.

Costella, M.F., Saurin, T.A., de Macedo Guimarães, L.B., 2009. A method for assessing health and safety management systems from the resilience engineering perspective. Safety

Science 47, 1056-1067.

Dekker, S., 2001. Follow the procedures or survive. Human Factors and Aerospace Safety 1(4), 381-385.

Dekker, S., 2003. Failure to adapt or adaptations that fail: Contrasting models on procedures and safety. Applied Ergonomics 34, 233-238.

DePoy, E., \& Gitlin, L. N., 2005. Introduction to Research: Understanding and Applying Multiple Strategies (3rd ed.). Elsevier, St. Loius, MO.

Dien, Y., 1998. Safety and application of procedures, or 'how do 'they' have to use operating procedures in nuclear power plants?’ Safety Science 29, 179-187.

Emerson, R. M., Fretz, R. L., \& Shaw, L. L., 1995. Writing Ethnographic Fieldnotes. The University of Chicago Press, Chicago.

Fetterman, D. M., 1989. Ethnography Step by Step. Sage, Newbury Park, CA.

Geertz, C., 1975. The Interpretation of Cultures. Hutchinson \& Co., London.

Gittleman, J.L., Gardner, P.C., Haile, E., Sampson, J.M., Cigularov, K.P., Ermann, E.D., Stafford, P. \& Chen, P.Y., 2010. [Case study] CityCenter and Cosmopolitan construction projects, Las Vegas, Nevada: Lessons learned from the use of multiple sources and mixed methods in a safety needs assessment. Journal of Safety Research 41, 263-281.

Grote, G., Weichbrodt, J.C., Günter, H., Zala-Mezö, E. \& Künzle, B., 2009. Coordination in high-risk organizations: The need for flexible routines. Cognition, Technology and Work 11, 17-27.

Hale, A. \& Borys, D., 2010.Working to rule, or working safely? Paper presented at 
WorkingonSafety.net, 7-10 September, Norway.

Hale, A.R. \& Swuste, P., 1998. Safety rules: Procedural freedom or action constraint? Safety Science 29, 163-177.

Hale, A.R., Heijer, T. \& Koornneef, F., 2003. Management of safety rules: The case of railways. Safety Science Monitor 7(1), Article III-2.

Harms-Ringdahl, L., 2004. Swedish case study - Safety Rule Management in Railways (Report). Stockholm, Sweden: Insititute for Risk Management and Safety Analysis.

Haslam, R.A., Hide, S.A., Gibb, A.G.F., Gyi, D.E., Pavitt, T., Atkinson, S. \& Duff, A.R., 2005. Contributing factors in construction accidents. Applied Ergonomics 36, 401415.

Hoonakker, P., Loushine, T., Carayon, P., Kallman, J., Kapp, A. \& Smith, M.J., 2005. The effects of safety initiatives on safety performance: A longitudinal study. Applied Ergonomics 36, 461-469.

Howell, G.A., Ballard, G., Abdelhamid, T.S. \& Mitropoulos, P., 2002. Working near the edge: A new approach to construction safty. Proceeding IGLC, $10^{\text {th }}$ August, Gramado, Brazil.

Iszatt-White, M., 2007. Catching them at it: An ethnography of rule violation. Ethnography 8(4), 445-465.

Kartam, N.A., Flood, I. \& Koushki, P., 2000. Construction safety in Kuwait: Issues, procedures, problems, and recommendations. Safety Science 36, 163-184.

Knudsen, F., 2009. Paperwork at the service of safety? Workers' reluctance against written procedures exemplified by the concept of 'seamanship'. Safety Science 47, 295-303.

Larsen, L. \& Hale, A.R. Safety rule management in railways. Proceedings of the European Transport Conference 2004. Strasbourg, 4-6 October. Association fro European Transport.

Laurence, D., 2005. Safety rules and regulations on mine sites - The problem and a solution. Journal of Safety Research 36(1), 39-50.

Loosemore, M. \& Andonakis, N., 2007. Barriers to implementing OHS reforms - The experiences of small subcontractors in the Australian Construction Industry. International Journal of Project Management 25, 579-588.

Loukopoulou, L., 2008. Pilot error: Even skilled experts make mistakes. Paper presented at WorkingonSafety.net, Crete, Greece, $30^{\text {th }}$ September $-3^{\text {rd }}$ October.

McDonald, M.A., Lipscomb, H.J., Bondy, J. \& Glazner, J., 2009. “Safety is everyone’s job:” The key to safety on a large university construction site. Journal of Safety Research 40, 53-61.

McDonald, N., Corrigan, S., Daly, C. \& Cromie, S., 2000. Safety management systems and safety culture in aircraft maintenance organisations. Safety Science (34(1-3), 151-176.

McDonald, R., Waring, J. \& Harrison, S., Walshe, K. \& Boaden, R., 2005. Rules and guidelines in clinical practice: A qualitative study in operating theatres of doctors' and nurses' views. Quality and Safety in Health Care 14, 290-294.

McDonald, R., Waring, J. \& Harrison, S., 2006. Rules, safety and the narrativisation of indentity: A hospital operating theatre case study. Sociology of Health \& Illness 28(2), 178-202.

Marchand, A., Simard, M., Carpentier-Roy, M. \& Ouellet, F., 1998. From a unidimensional to a bidimensional concept and measurement of workers' safety behavior. Scandinavian Journal of Work \& Environmental Health 24(4), 293-299.

Miles, M. B., \& Huberman, A. M., 1994. Qualitative Data Analysis (2nd ed.). Sage, Thousand Oaks, CA.

Mitropoulos, P.T. \& Cupido, G., 2009a. The role of production and teamwork practices in construction safety: A cognitive model and an empirical case study. Journal of Safety 
Research 40, 265-275.

Mitropoulos, P. \& Cupido, G., 2009b. Safety as an emergent property: Investigation into the work practices of high-reliability framing crews. Journal of Construction Engineering and Management 135(5), 407-415.

Mitropouslos, P., Abdelhamind, T.S. \& Howell, G.A., 2005. Systems model of construction accident causation. Journal of Construction Engineeringand Management 131(7), 816825.

Mohamed, S., Ali, T.H. \& Tam, W.Y.V., 2009. National culture and safe work behavior of construction workers in Pakistan. Safety Science 47, 29-35.

Nathanael, D. \& Marmaras, N., 2008. On the development of work practices: A constructivist model.

Theoretical Issues in Ergonomics Science 9(5), 359-382.

NOHSC., 2005. National Standard for Construction Work [NOHSC:1016 (2005)]. Commonwealth of Australia, Canberra.

Neuman, W. L., 2003. Social Research Methods: Qualitative and Quantitative Approaches (5th ed.). Allyn \& Bacon, Boston, MA.

Occupational Health and Safety Regulations 2007. S.R. No. 54/2007. Government of Victoria.

OFSC, 2010. Guidance for producing Safe Work Method Statements (SWMS). Department of Education, Employment and Workplace Relations, Canberra.

OGSC, 2007. Cochrane Systematic Review on the Efficacy of Interventions to Prevent Injuries in the Construction Industry. Department of Education, Employment and Workplace Relations, Canberra.

OHSA, 2002. Job Hazard Analysis. U.S. Department of Labor.

Rochlin, G.L., 1999. Safe operation as a social construct. Ergonomics 42(11), 1549-1560.

Rozenfeld, O., Sacks, R., Rosenfeld, Y. \& Baum, H., 2010. Construction job safety analysis. Safety Science 48, 491-498.

Safe Work Australia, 2010. Construction. Commonwealth of Australia, Barton, ACT.

Safe Work Australia, 2010. Key Work Health and Safety Statistics, Australia. Commonwealth of Australia, Barton, ACT.

Saurin, T.A., Formoso, C.T. \& Cambraia, F.B., 2008. An analysis of construction safety best practices from a cognitive systems engineering perspective. Safety Science 46, 11691183.

Silverman, D., 2010. Doing qualitative research ( $3^{\text {rd }}$ Ed.). Sage. Thousand Oaks, California.

Simard, M. \& Marchand, A., 1997. Workgroups' propensity to comply with safety rules: The influence of micro-macro organisational factors. Ergonomics 40(2), 172-188.

Smircich, L. \& Morgan, G., 1982. Leadership: The management of meaning. The Journal of Applied Behavioral Science 18(3), 257-273.

Suraji, A., Duff, A.R. \& Peckitt, S.J., 2001. Development of causal model of construction accident causation. Journal of Construction Engineering and Management 127(4), 337-344.

Teo, E.A.L., Ling, F.Y.Y. \& Chong, A.F.W., 2005. Framework for project managers to manage construction safety. International Journal of Project Management 23, 329-341.

Thackaberry, J.A., 2004. "Discrsive opening” and closing in organizational self-study: Culture as trap and tool in wildland firefighting safety. Management Communication Quarterly 17(3), 319-359.

Törner, M. \& Pousette, A., 2009. Safety in construction - a comprehensive description of the characteristics of high safety standards in construction work, from the combined perspective of supervisors and experienced workers. Journal of Safety Research 40, 399-409. 
Turner, N. \& Gray, G.C., 2009. Socially constructing safety. Human Relations 62(9), 12591266.

Weick, K.E., 1993. The collapse of sensemaking in organizations: The Mann Gulch disaster. Administrative Science Quarterly 38, 628-652.organizations: The Mann Gulch disaster. Administrative Science Quarterly 38, 628-652.

Weick, K.E.,1995. Sensemaking in organizations. Sage. Thousand Oaks, California.

WorkCover NSW, 2001. Priority Issues for Construction Reform: Safely Building New South Wales - Report Summary. WorkCover NSW, Sydney.

Wright, P. \& McCarthy, J., 2003. Analysis of procedure following as concerned work. In E. Hollnagel (Ed.), Handbook of Cognitive Task Design (pp. 679-700). SRC Press, Hoboken.

Zhou, Q., Fang, D. \& Wang, X., 2008. A method to identify strategies for the improvement of human safety behavior by considering safety climate and personal experience. Safety Science 46, 1406-1419. 